\section{REVISTA BRASILEIRA DE QUALIDADE DE VIDA}

\title{
Análise diagnóstica da Qualidade de Vida no Trabalho em uma indústria de embalagens
}

\section{Diagnostic analysis of the Quality of Working Life in a packaging industry}

\author{
Gabriela Mantovani Teixeira \\ Centro Universitário das Faculdades Associadas de Ensino - UNIFAE- São João da Boa Vista - Brasil \\ gaby_1.m.1@ hotmail.com \\ Valdete Maria Ruiz \\ Centro Universitário das Faculdades Associadas de Ensino - UNIFAE- São João da Boa Vista - Brasil \\ valdete@fae.br
}

\section{RESUMO}

OBJETIVO: Analisar o nível da Gestão da Qualidade de Vida no Trabalho (QVT) na estrutura de uma organização industrial, relacionando-a com variáveis do contexto e com a percepção da QVT pelos seus colaboradores.

MÉTODOS: A pesquisa foi realizada em uma indústria de embalagens de papelão, localizada no interior do Estado de São Paulo. Para a coleta de dados utilizou-se entrevista com a gerente de Recursos Humanos, sessões de observação dos setores de trabalho e aplicação coletiva do questionário QWLQ-bref, em uma amostra correspondente a 50,0 \% dos colaboradores dos setores administrativo e operacional, selecionados por acessibilidade. Foi utilizado o procedimento da análise de conteúdo nas entrevistas, registro cursivo das observações e cálculos de médias e percentuais dos 60 questionários considerados válidos $(48,9 \%$ do universo da pesquisa), os quais foram classificados por níveis de satisfação com a QVT, segundo critérios dos autores do QWLQ-bref.

RESULTADOS: A entrevista indicou que a organização estava no nível Operacional da Gestão da QVT. Nas observações foram evidenciadas condições de desconforto ambiental e limitações na interação, comunicação e participação dos funcionários. No QWLQ-bref obtevese média de 3,63 (72,6\%) na avaliação da QVT como fator Global. O domínio Físico/Saúde teve média 3,70 (74,0\%), o domínio Psicológico 3,56 (71,2\%), o domínio Pessoal 3,86 $(77,2 \%)$ e o domínio Profissional 3,47 (69,4\%). Todas as avaliações dos domínios da QVT pelos participantes foram consideradas satisfatórias.

CONCLUSÕES: A percepção da QVT dos colaboradores da indústria pesquisada é satisfatória e congruente com dados encontrados em estudos similares, embora o nível da Gestão da QVT tenha sido considerado menos positivo e algumas variáveis do contexto tenham se mostrado limitadoras. Os resultados obtidos reforçam a importância das avaliações pessoais dos colaboradores na análise diagnóstica da QVT e sugerem que estudos posteriores avancem nessa direção.

PALAVRAS-CHAVE: Avaliação. Gestão. Qualidade de Vida no Trabalho. QWLQ-bref. 


\section{ABSTRACT}

OBJECTIVE: To analyze the level of Management of the Quality of Work Life (QWL) in the structure of industrial organization, relating it to context variables and the perception of QWL by its contributors.

METHODS: The research was conducted in a cardboard packaging industry, located in the State of São Paulo. For data collection it was used an interview with the Human Resources manager, observation sessions of work sectors and collective application of QWLQ-bref questionnaire in a sample corresponding to $50.0 \%$ of the employees of the administrative and operational sectors, selected by accessibility. It was used the procedure of content analysis in interviews, cursive record observations and calculations of averages and percentages of 60 considered valid questionnaires (48.9\% of the survey), which were classified by levels of satisfaction with QWL, according criteria from the authors of QWLQ-bref.

RESULTS: The interview indicated that the organization was in operational level of QLW management. In the observation sessions were evidenced environmental discomfort conditions and constraints on interaction, communication and employee participation. QWLQ-bref obtained an average of $3.63(72.6 \%)$ in the evaluation of QWL as a Global factor. The Physical / Health domain averaged 3.70 (74.0\%), the Psychological domain 3.56 (71.2\%), the Personal domain $3.86(77.2 \%)$ and the Professional domain 3.47 (69, 4\%). All QVT evaluation of the domains from participants were considered satisfactory.

CONCLUSIONS: The QWL perception of employees in this researched industry is satisfactory and consistent with data found in similar studies, although the level of the Management of the QWL has been considered less positive and some context variables have been shown to be limiting. The results reinforce the importance of personal evaluations of employees in the diagnostic analysis of QWL and suggest that further studies advance in this direction.

KEYWORDS: Evaluation. Management. Quality of Work Life. QWLQ-bref.

\section{Introdução}

O conceito de Qualidade de Vida no Trabalho (QVT) é complexo, polissêmico e, por isso, tem sido definido de diferentes formas, desde que essa expressão foi criada, na segunda metade do século XX. Mas, embora não haja unanimidade nas definições, existe convergência para dois pontos. O primeiro vai no sentido de que a QVT seja representada como uma ramificação da Qualidade de Vida (QV) e o segundo indica que a QVT refere-se a um conjunto de fatores (objetivos e subjetivos) que incorporam satisfação do colaborador em sua atividade laboral, humanizam as situações relacionadas ao trabalho e levam à obtenção de maior produtividade e qualidade por parte da organização (RUIZ, 2007; SILVA; PEDROSO; PILATTI, 2010; PEDROSO; PILATTI, 2010). De fato, é reconhecido que as empresas que implantam um programa de QVT conseguem maior eficácia e produtividade, ao mesmo tempo em que atendem às necessidades básicas de seus trabalhadores, entre as quais o aumento do seu bem-estar e participação nas decisões e problemas do trabalho (CHAMON, 2011; LIMONGI-FRANÇA, 2010; SANT’ANNA; KILIMNIK; MORAES, 2011).

Em sua revisão da literatura, Medeiros (2007) reuniu dez dimensões/fatores que estão presentes nas diferentes concepções teóricas (desde as mais clássicas até as mais modernas) a respeito de QVT: execução do trabalho; autorrealização; equidade no trabalho; relação com a organização; adequação da remuneração; relação chefe-subordinado; efetividade da comunicação; relação trabalho e vida pessoal; efetividade dos processos de trabalho. Esse conjunto, bem como os respectivos indicadores elencados pelo autor, ilustra o quanto é 
complexo e amplo o leque de fatores que estão presentes nas concepções sobre o tema. Vale destacar que boa parte dos fatores advém do trabalho pioneiro de Walton (1973), ainda hoje considerado o modelo mais completo para se avaliar e intervir sobre a QVT (PEDROSO; PILATTI, 2010; RUIZ, 2007).

Analisando os avanços e limitações, e também tecendo críticas aos estudos sobre o tema, Sant'Anna, Kilimnik e Moraes (2011) consideram que: (a) existe predominância de estudos quantitativos, e que uma complementação com estudos de natureza qualitativa seria desejável para melhor compreensão da dinâmica das situações estudadas; (b) a maioria dos trabalhos limita-se a verificar o nível em que a QVT se encontra, deixando para segundo plano a análise da complexidade das variáveis que influenciam sua configuração; (c) há reduzido número de estudos alicerçados no entendimento da QVT sob a percepção dos próprios indivíduos pesquisados e um caráter elitista de diversos estudos e intervenções nesse campo, dado seu foco, durante longo tempo, na alta e média administrações, em especial, de grandes corporações; (d) há que se salientar a busca pela compreensão da QVT sob uma perspectiva menos diagnóstica e mais relacionada à noção de gestão.

É sob esse último ponto de ponto de vista que, nas organizações contemporâneas, cada vez mais, discute-se a importância de uma adequada Gestão da Qualidade de Vida no Trabalho (GQVT). Segundo Limongi-França (2010), a GQVT deve ser encarada como uma competência estratégica-gerencial, e necessita da utilização de diferentes conceitos, ferramentas, participação e indicadores visando melhor equilíbrio da vida pessoal e profissional, com autoestima valorizada, hábitos saudáveis, política de equidade, justiça social e sustentabilidade.

Entretanto, há múltiplas possibilidades de enfoque na GQVT, dependendo da abrangência com que o próprio termo QVT seja considerado e também das realidades/possibilidades de cada organização. Assim, podem ser definidos três níveis e implicações da GQVT na estrutura das empresas:

Gestão estratégica de QVT (GE-QVT) quando é declarada na missão e política da empresa, juntamente com a imagem corporativa.

Gestão Gerencial de QVT (GG-QVT) quando aparece como responsabilidade e atribuição dos líderes e chefes das áreas e departamentos específicos no segundo ou terceiro nível hierárquico, com ênfase em objetivos, metas e produtividade organizacional.

Gestão Operacional de QVT quando há ações específicas - aleatórias, reativas ou planejadas - visando ao bem-estar e à conscientização de novas práticas de estilo de vida para as pessoas da empresa, mas não alinhadas aos propósitos de competitividade ou otimização do gerenciamento, da produtividade e da performance para o trabalho (LIMONGI-FRANÇA, 2001, p. 244).

A fim de que a GQVT se torne cada vez mais estratégica nas organizações, Ogata e Simurro (2009) recomendam adotar quatro etapas, de forma sequencial e cíclica: pesquisa e avaliação diagnóstica; planejamento; lançamento e implementação; avaliação de resultados. Destacam a importância da primeira etapa, considerando que ela pode durar de três meses a um ano, e que se deve, inicialmente, analisar o grau de prontidão para o lançamento de ações relacionadas à QVT. Isto porque, apesar de ser esta uma tendência seguida pelos modelos mais modernos de gestão de pessoas, alguns líderes empresariais ainda têm dificuldade de entender como incorporar programas e ações visando à saúde, bem-estar e QV à estratégia da organização, como afirmam os mesmos autores. Na sequência, segundo Ogata e Simurro (2009), é fundamental que a organização defina um gestor, ou seja, um responsável por aglutinar as ações e programas de QVT. Depois disso, deve ser constituído um comitê de 
gestão, a partir do que deve começar a avaliação diagnóstica propriamente dita, utilizando-se de indicadores e instrumentos pertinentes.

A importância de uma adequada análise diagnóstica, visando à adoção de programas na linha de QVT, também é destacada em modelos de GQVT propostos por outros autores, entre os quais Fernandes (1996), na chamada Auditoria Operacional de Recursos Humanos para a melhoria da QVT (AORH), e Limongi-França (2001), que considera seis etapas para a implantação de projetos de QVT: conscientização, diagnóstico; capacitação, criação de planos de ação, implantação e avaliação. Em geral, estes autores, assim como Ogata e Simurro (2009), salientam que para uma boa análise diagnóstica sejam utilizados vários instrumentos, indicadores e estratégias para a coleta de dados sobre a situação da QVT (de preferência baseados em suas diferentes concepções teóricas). Entre estes estão: registros e índices (sobre rotatividade, absenteísmo, presenteísmo, doenças ocupacionais e reclamações trabalhistas, por exemplo), observações nos locais onde se realiza cada tipo de trabalho para se obter indicadores sobre as condições físicas, ambientais e organizacionais (velocidade, ritmo, divisão de tarefas, relacionamentos interpessoais, entre outros) e informações internas ou externas à organização (como clima organizacional e imagem na comunidade).

Quanto a instrumentos para a avaliação da QVT, são vários os disponíveis na literatura atual: roteiros de entrevistas, questionários e levantamentos como o Índice de Kertesz e Kerman, o Inventário de Qualidade de Vida Profissional 35, a Escala de Percepção dos indivíduos quanto à QVT e os questionários de Qualidade de Vida no Trabalho QWLQ-78 e o QWLQ-bref (CHEREMETA et al., 2011; FERNANDES, 1996; LIMONGI-FRANÇA, 2010; MEDEIROS; OLIVEIRA, 2011; OGATA; SIMURRO, 2009; REIS JÚNIOR; PILATTI; PEDROSO, 2011).

O Quality of Working Life Questionnaire - QWLQ-78, questionário de QVT, é um instrumento desenvolvido no Brasil por Reis Júnior, em 2008, com a mesma metodologia utilizada pelo Grupo WHOQOL para a criação do WHOQOL-100 (um dos instrumentos mais empregados, atualmente, para se avaliar a QV). Está baseado nos modelos mais difundidos de QVT, e abrange os seguintes fatores ou domínios da QVT: Físico/Saúde, Psicológico, Pessoal e Profissional. O domínio Físico/Saúde aborda aspectos relacionados à saúde, doenças relacionadas ao trabalho e hábitos dos colaboradores de uma dada organização; o domínio Psicológico refere-se aos aspectos relacionados à satisfação pessoal, motivação no trabalho e autoestima dos colaboradores; o domínio Pessoal aborda os aspectos familiares, crenças pessoais e religiosas e aspectos culturais que influenciem o trabalho; o domínio Profissional refere-se aos aspectos que influenciam, de maneira global, a percepção de QVT (REIS JÚNIOR; PILATTI; PEDROSO, 2011).

Posteriormente, Cheremeta et al. (2011) criaram uma versão abreviada do QWLQ-78, a qual possibilita mais rapidez na sua aplicação e avaliação: o QWLQ-bref. Provavelmente por essa razão, esse instrumento venha sendo cada vez mais utilizado em estudos brasileiros sobre QVT, como o de Medeiros (2012), que comparou a QV fora do trabalho (avaliada pelo WHOQOL-abreviado) e a QVT de profissionais de saúde de um município do Estado do Rio Grande do Sul. No estudo em questão, a QV apresentou-se em patamares superiores à QVT, sendo que esta alcançou menores escores nos domínios Físico/Saúde e Profissional.

Vale destacar que no estudo de validação do QWLQ-78, realizado com trabalhadores da indústria, comércio e serviços de cidades do Estado do Paraná, as menores médias também foram encontradas nos domínios Profissional e Físico/Saúde (REIS JÚNIOR; PILATTI; PEDROSO, 2011).

Tendo em vista o exposto, o presente estudo teve como objetivo analisar o nível da GQVT na estrutura de uma organização industrial, relacionando-o com variáveis do contexto e com a percepção da QVT pelos seus colaboradores. 


\section{Métodos}

Para atender aos objetivos do estudo foi realizada uma pesquisa que pode ser caracterizada como de campo, descritiva e analítica (VERGARA, 2003), com o uso de métodos e técnicas quantitativos e qualitativos, em uma indústria localizada na região leste do Estado de São Paulo, cujo ramo de atividade é a fabricação de embalagens de papelão ondulado e gráfica off-set. À época em que foi realizada (entre março e maio de 2012), a indústria contava com 133 colaboradores diretos (03 na gerência, 56 no setor administrativo e 74 no setor operacional).

Os procedimentos de coleta e análise de dados foram divididos em três fases, sendo a primeira a realização de uma entrevista semiestruturada com a gerente do departamento de Recursos Humanos (RH). Esta entrevista baseou-se num roteiro de perguntas previamente elaborado pelas autoras do estudo, com base em Limongi-França (2001), visando identificar o enfoque sobre a QVT, assim como o nível e implicações da GQVT na organização. As respostas foram anotadas por duas entrevistadoras para posterior análise de conteúdo, que foi realizada segundo as indicações de Bardin (2010).

$\mathrm{Na}$ segunda fase foi utilizada a técnica de observação de campo, com registro cursivo (FAGUNDES, 2006), focalizando-se as variáveis: condições de trabalho (iluminação, ventilação, temperatura, agentes químicos, poeira, outros riscos ambientais, uso de uniformes, equipamentos de proteção individuais - EPIs, ritmo e velocidade do trabalho); comunicação (tipo e frequência); relacionamento interpessoal; relacionamento chefe-subordinado; outros aspectos considerados importantes sob o ponto de vista da QVT dos trabalhadores. Essas observações tiveram a duração média de uma hora em cada setor de trabalho, em horários distintos, e também foram realizadas por duas observadoras para posterior análise dos comportamentos e variáveis da situação e do contexto.

A terceira e última fase constou da aplicação do instrumento QWLQ-bref para a avaliação dos domínios e dimensões da QVT, em uma amostra de 65 indivíduos (50,0\% dos colaboradores dos setores administrativo e operacional da empresa, excluindo-se seus gerentes), constituída por acessibilidade. A aplicação foi feita de forma coletiva, em uma sala da própria indústria. A estratégia de coleta de dados levou em conta as escalas de horários dos funcionários envolvidos. Estes manisfestaram sua concordância em participar do estudo após serem informados de que se tratava de um trabalho acadêmico e de que suas respostas seriam mantidas em sigilo, sendo-lhes garantido o anonimato.

O questionário QWLQ-bref (CHEREMETA et al., 2011) é composto de 20 questões para avaliação dos domínios Físico/Saúde, Psicológico, Pessoal e Profissional da QVT, além da QVT como fator global. As questões são apresentadas em uma escala do tipo Likert de cinco alternativas, em que o ponto 1 representa uma resposta muito negativa e o ponto 5 representa uma resposta muito positiva.

Após a aplicação dos questionários QWLQ-bref foi feita a tabulação das respostas em planilha Microsoft Excell, com cálculos das médias e percentuais por questões e domínios da QVT. Importante destacar que cinco questionátios foram excluídos da amostra por conterem rasuras ou não ter atingido o percentual mínimo de $80 \%$ de questões respondidas em cada domínio, como recomendam seus autores. Para a classificação dos resultados seguiram-se as indicações de Cheremeta et al. (2011) que, por sua vez, utilizaram a mesma escala de classificação em níveis (muito insatisfatório, insatisfatório, neutro, satisfatório e muito satisfatório) proposta para o QWLQ-78 (REIS JÚNIOR; PILATTI; PEDROSO, 2011). 


\section{Resultados e Discussão}

Segundo as informações obtidas na primeira fase da pesquisa, por meio da entrevista com a Gerente de RH, a empresa sempre busca agregar, em seus sistemas de trabalho, técnicas e inovações que favoreçam a QVT, pois entende que seus colaboradores trabalharão melhor se tiverem uma boa QVT. Procuram favorecê-la com palestras, consultorias externas e atuação da Comissão Interna de Prevenção de Acidentes (CIPA). Essas iniciativas são, em geral, de responsabilidade do Departamento de $\mathrm{RH}$, e ocorrem, mais frequentemente, por ocasião da Semana Interna de Prevenção de Acidentes (SIPAT). Nas iniciativas são tratados temas relativos à saúde, higiene e segurança, sendo que estas não costumam ser sistematicamente planejadas com base nas necessidades e expectativas dos colaboradores. Tampouco são avaliadas pela organização.

Constatou-se a existência de interesse e prontidão da empresa pesquisada para implementar ações relativas à QVT, mas que, segundo o critério de Limongi-França (2001), o nível de GQVT seria classificado como Gestão Operacional, visto que as ações desenvolvidas visavam, mais especificamente, ao bem-estar, à saúde e à segurança das pessoas da empresa, sem estarem alinhadas aos propósitos de competitividade ou otimização do gerenciamento, da produtividade e da performance para o trabalho. No entanto, para se chegar a uma conclusão mais segura sobre isso, seria desejável fazer mais ampla investigação, utilizando-se outras fontes de dados, como recomendam Fernandes (1996), Limongi-França (2010) e Ogata e Simurro (2009).

As observações realizadas nos locais de trabalho, que constituíram a segunda fase do estudo, permitiram verificar que havia pouca interação entre os funcionários do setor operacional, visto que cada um desempenhava suas tarefas de forma independente dos demais. Nos locais onde havia máquinas que produziam ruídos, a comunicação verbal era muito restrita. Já nos locais onde havia bancadas manuais, assim como no setor administrativo, as interações eram relativamente mais frequentes, sendo que as comunicações verbais registradas incluíam assuntos de trabalho, assuntos pessoais, brincadeiras e risos. A interação estabelecida entre chefes e subordinados dos três setores ocorria rapidamente, em geral para a transmissão de ordens de serviço e demais orientações técnicas.

Com relação às condições ambientais dos locais de trabalho, observou-se que, em todos, havia iluminação e ventilação adequadas, além de facilidade no acesso aos bebedouros e banheiros. As tarefas realizadas pelos funcionários do setor operacional exigiam ritmo intenso e movimentos repetitivos, havendo marcada divisão de trabalho. Em um dos locais do setor operacional existia odor intenso de tinta e plástico, oriundos das matérias-primas.

Sendo assim, as observações evidenciaram restrita interação e comunicação, tanto entre colegas de trabalho de um mesmo setor, quanto entre eles e seus chefes e entre diferentes setores. A divisão de trabalho, a limitada interdependência entre as tarefas, o ruído e a presença de substâncias químicas (no caso do setor operacional) são fatores limitadores da QVT, tendo-se em vista o que indicam vários dos modelos teóricos sobre o tema, como o de Walton (1973).

Dos 60 questionários QWLQ-bref aplicados e considerados válidos na terceira etapa da pesquisa, $17(28,3 \%)$ eram de colaboradores do setor administrativo e $43(71,7 \%)$ do setor operacional. No total, a amostra dessa fase da pesquisa correspondeu a 48,9\% do efetivo de funcionários da empresa (excluídos os de nível gerencial). Foi constituída de 36 (60,0\%) homens e $24(40,0 \%)$ mulheres, com idades entre 18 e 59 anos, escolaridade desde Ensino Fundamental incompleto até Pós-graduação lato sensu e tempo de atuação na empresa entre 02 meses e 13 anos.

As médias obtidas em cada domínio e questão do QWLQ-bref junto a essa amostra são apresentadas na Tabela 1 , onde se pode verificar que a satisfação com a QVT, como fator 
global obteve a média 3,63 (72,6\%), índice considerado satisfatório, de acordo com o critério de classificação adotado.

Tabela1 - QVT: Médias obtidas em cada domínio e questão do QWLQ-bref

\begin{tabular}{|c|c|}
\hline Questões por Domínio & Média \\
\hline Físico/Saúde & 3,70 \\
\hline Q4 - Em que medida você avalia o seu sono? & 3,79 \\
\hline Q8 - Em que medida algum problema com o sono prejudica seu trabalho? & 4,25 \\
\hline Q17 - Suas necessidades fisiológicas básicas são satisfeitas adequadamente? & 3,60 \\
\hline Q19-Em que medida você se sente confortável no ambiente de trabalho? & 3,62 \\
\hline Psicológico & 3,56 \\
\hline Q2 - Em que medida você avalia sua motivação para trabalhar? & 3,55 \\
\hline Q5 - Como você avalia sua liberdade de expressão no seu trabalho? & 3,25 \\
\hline Q9 - Em que medida você avalia o orgulho pela sua profissão? & 3,90 \\
\hline Pessoal & 3,86 \\
\hline Q6 - Você se sente realizado com o trabalho que faz? & 3,85 \\
\hline Q10 - Como você avalia a qualidade da sua relação com seus superiores e/ou subordinados? & 3,46 \\
\hline Q11 - Em que medida sua família avalia o seu trabalho? & 4,34 \\
\hline Q15 - Em que medida você é respeitado pelos seus colegas e superiores? & 76,6 \\
\hline Profissional & 3,47 \\
\hline Q1 - Como você avalia a sua liberdade para criar coisas novas no trabalho? & 3,35 \\
\hline Q3 - Como você avalia a igualdade de tratamento entre os funcionários? & 3,41 \\
\hline Q7 - Em que medida você possui orgulho da organização na qual trabalha? & 3,66 \\
\hline Q12 - Em que medida você está satisfeito com o seu nível de participação nas decisões da empresa? & 2,98 \\
\hline Q13 - Você está satisfeito com o seu nível de responsabilidade no trabalho? & 3,79 \\
\hline Q14 - Você se sente satisfeito com os treinamentos dados pela organização? & 3,35 \\
\hline Q16 - Você se sente satisfeito com a variedade de tarefas que realiza? & 3,60 \\
\hline Q18 - Como você avalia o espírito de camaradagem no seu trabalho? & 3,55 \\
\hline $\begin{array}{l}\text { QVT como fator Global } \\
\text { Q20 - O quanto você está satisfeito com a sua qualidade de vida no trabalho? }\end{array}$ & 3,63 \\
\hline
\end{tabular}

Destaque-se que esse resultado é praticamente igual ao apresentado por Medeiros (2012), com a aplicação do QWLQ-bref em profissionais de saúde do Rio Grande do Sul $(3,65)$, e pouco superior ao apresentado no estudo de Reis Júnior, Pilatti e Pedroso (2011), com a aplicação do QWLQ-78 em trabalhadores do Paraná $(3,53)$.

O domínio Físico/Saúde obteve média 3,70 (74,0\%) - resultado também considerado satisfatório, mas ligeiramente superior aos obtidos nos estudos de Reis Júnior, Pilatti e Pedroso (2011) e Medeiros (2012): o primeiro com média 3,49 e o segundo com média 3,61. Em relação aos demais domínios, foi o segundo com melhor avaliação. Todas as questões que o compõem são consideradas satisfatórias, com exceção da Q-8 (Em que medida algum problema com o sono prejudica seu trabalho), que atingiu o nível de classificação muito satisfatório $(85,0 \%)$. As questões onde foram obtidos menores médias nesse domínio foram a questão 17 (satisfação das necessidades fisiológicas básicas) e a questão 19 (conforto no ambiente de trabalho), o que pode ser decorrência do ritmo intenso de trabalho, da presença de ruído constante e de produtos químicos nos setores operacionais, conforme foi observado.

O domínio Psicológico foi o terceiro mais bem avaliado pelos colaboradores que participaram da pesquisa, apresentando média 3,56 (71,2\%), resultado igualmente classificado como satisfatório. Neste domínio, a média obtida por Reis Júnior, Pilatti e Pedroso (2011) foi 
muito semelhante $(3,51)$ e a obtida por Medeiros (2012) foi superior $(3,85)$. Todas as questões do domínio classificam-se no nível satisfatório, merecendo destaque a Q5 (Como você avalia sua liberdade de expressão no trabalho) na qual foi obtida a menor média $(3,25)$ em relação a todas as demais do QWLQ-bref.

No domínio Pessoal, da mesma forma que nos estudos de Reis Júnior, Pilatti e Pedroso (2011) e Medeiros (2012), foi obtido o maior índice de QVT da amostra. A média alcançada foi 3,86 (77,2\%), o que representa que a QVT dos pesquisados encontra-se em nível satisfatório, sob o ponto de vista pessoal. Esse resultado é intermédiário entre os obtidos pelo primeiro e pelo segundo estudos citados (3,74 e 4,04, respectivamente). Vale sublinhar que nesse domínio encontrou-se a maior média por questão na Q11 (Em que medida sua família avalia o seu trabalho). Essa média $(4,34)$ atingiu o nível muito satisfatório $(86,8 \%)$, de acordo com a escala adotada, e indica que os participantes têm, em suas famílias, um ponto favorecedor de sua QVT, ponto considerado importante segundo a literatura (WALTON, 1973; LIMONGI-FRANÇA, 2010).

Também de forma semelhante aos estudos de Reis Júnior, Pilatti e Pedroso (2011) e Medeiros (2012), o domínio Profissional foi o que obteve o menor índice de QVT dentre os quatro domínios avaliados. Obteve-se o resultado médio de 3,47 (69,4\%), considerado satisfatório, de acordo com o critério adotado neste estudo, e bem próximo aos dos estudos citados (3,38 no primeiro e 3,57 no segundo). Cumpre notar que a Q12 (Em que medida você está satisfeito com o seu nível de participação nas decisões da empresa) foi a questão que obteve o menor escore comparado a todos os demais do instrumento utilizado para avaliar a QVT $(2,98)$, resultado esse que, embora ainda seja considerado satisfatório $(59,6 \%)$, aproxima-se mais do nível neutro da escala de classificação adotada. A literatura indica que a participação nas decisões e problemas do trabalho é um aspecto importante a ser considerado na GQVT das empresas, já que se refere a uma necessidade psicológica do ser humano (CHAMON, 2011; LIMONGI-FRANÇA, 2010; SANT’ANNA; KILIMNIK; MORAES, 2011). Sendo assim, o resultado obtido na Q-12 reforça a importância dessa dimensão para favorecer a QVT no domínio Profissional.

Outro aspecto que merece análise é que, se comparados os dados obtidos nos quatro domínios da QVT, verifica-se que houve variação de quase oito pontos percentuais entre o mais bem avaliado (Pessoal) e o menos bem avaliado (Profissional). Essa diferença pode ser considerada importante, na medida em que a GQVT, como uma competência estratégica das organizações, visa ao melhor equilíbrio da vida pessoal e profissional (entre outros aspectos), conforme salientam Limongi-França (2010) e Medeiros (2007), além de outros autores.

Em síntese, os resultados obtidos indicam que a percepção da QVT dos colaboradores da indústria pesquisada é satisfatória e congruente com dados encontrados em estudos similares, embora o nível da GQVT tenha sido considerado como o da Gestão Operacional e algumas variáveis do contexto organizacional observadas tenham se mostrado limitadoras da QVT (ruído e presença de substâncias químicas nos locais de trabalho, acentuada divisão de trabalho com pouca interdependência entre tarefas, restrições na comunicação e interação entre os funcionários). Esses resultados vão ao encontro do que a literatura sobre o tema registra, ou seja, de que o conceito de QVT (assim como o de QV) engloba uma complexidade de fatores interrelacionados. E esses são tanto objetivos quanto (e, talvez, principalmente) subjetivos.

Em outras palavras, pode-se dizer que, mesmo não se encontrando condições tão satisfatórias do ponto de vista objetivo em relação à GQVT e às condições organizacionais, a avaliação pessoal (subjetiva) de cada indivíduo influencia de modo especial a QVT. Isso foi o que, em particular, demonstram os resultados do presente estudo.

\section{Conclusão}

A pesquisa realizada procurou atender às recomendações de estudiosos da área no sentido de buscar a utilização de métodos quantitativos e qualitativos para analisar a realidade 
estudada, sob o ponto de vista da análise diagnóstica da QVT como etapa fundamental para subsidiar sua gestão (GQVT) nas organizações.

As limitações do estudo são o reduzido número de fontes, indicadores e instrumentos utilizados, bem como, em relação à análise quantitativa e qualitativa dos dados obtidos. Assim, sugere-se que estudos posteriores avancem nessa direção, a fim de que possam levar a conclusões que ampliem as possibilidades de generalização.

Mesmo com essas restrições, o estudo evidenciou um aspecto fundamental a respeito da QVT, já bem sedimentado na literatura: o de que o aspecto psicológico é tão importante quanto os dados da realidade concreta. Isso porque os resultados das avaliações subjetivas realizadas pelos pesquisados situaram a QVT num nível satisfatório, enquanto que o nível de GQVT e algumas condições organizacionais não foram consideradas no mesmo nível pelas pesquisadoras. Assim, a presente pesquisa comprova que a análise diagnóstica da QVT não pode prescindir da percepção dos próprios trabalhadores, ou seja, do entendimento sobre o que, de fato, significa ter qualidade de vida em seu trabalho.

\section{Referências}

BARDIN, L. Análide de conteúdo. Lisboa: Edições 70, 2010.

CHAMON, E. M. Q. O. (org.). Qualidade de vida no trabalho. Rio de Janeiro: Brasport, 2011.

CHEREMETA, M. et al. Construção da versão abreviada do QWLQ-78: um instrumento de avaliação da qualidade de vida no trabalho. Revista Brasileira de Qualidade de Vida, v. 1, n. 3, p. 01-15, 2011.

FAGUNDES, A. J. F. M. Descrição, definição, e registro de comportamento. 14. ed. São Paulo: EDICON, 2006.

FERNANDES, E. C. Qualidade de vida no trabalho: como medir para melhorar. Salvador: Casa da Qualidade, 1996.

LIMONGI-FRANÇA, A. C. Treinamento e Qualidade de Vida. In: BOOG, G. G. Manual de Treinamento e Desenvolvimento. São Paulo: Makron Books/ABTD, 2001.

LIMONGI-FRANÇA, A. C. Qualidade de vida no trabalho - QVT: conceitos e práticas nas empresas da sociedade pós-industrial. 2. ed. 5. reimpr. São Paulo: Atlas, 2010.

MEDEIROS, C. H. Qualidade da vida dos profissionais do Sistema Único de Saúde (SUS) da Prefeitura Municipal de Alegrete (RS). 2012. 42 f. Trabalho de Conclusão de Curso (Especialização em Gestão Pública em Saúde) - Escola de Administração, Universidade Federal do Rio Grande do Sul, Alegrete. Disponível em: $<$ http://www.lume.ufrgs.br/bitstream/handle/10183/70374/000869993.pdf?sequence=1 >. Acesso em: 20 jun. 2013

MEDEIROS, J. P. Qualidade de vida no trabalho da EMATER-RN: validação de um instrumento síntese de pesquisa e diagnóstico. 2007. 158 f. Dissertação (Mestrado em Administração) - Programa de Pós-Graduação em Administração, Universidade Federal do Rio Grande do Norte, Natal. Disponível em: <ftp://ftp.ufrn.br/pub/biblioteca/est/bdtd/JassioPM.pdf>. Acesso em: 10 jun. 2013

OGATA, A.; SIMURRO, S. Guia prático de qualidade de vida: como planejar e gerenciar o melhor programa para a sua empresa. Rio de Janeiro: Elsevier, 2009. 
PEDROSO, B.; PILATTI, L. A. Revisão literária dos modelos clássicos de avaliação da qualidade de vida no Trabalho: um debate necessário. In: VILARTA, R.; GUTIERREZ, G. L.; MONTEIRO, M. I. (orgs.). Qualidade de vida: evolução dos conceitos e práticas no século XXI. Campinas, SP: Ipês, 2010.

REIS JÚNIOR, D. R.; PILATTI, L. A.; PEDROSO, B. Qualidade de vida no trabalho: construção e validação do questionário QWLQ-78. 2008. Revista Brasileira de Qualidade de Vida, Ponta Grossa, v. 3, n. 2, p. 1-12, jul./dez. 2011.

RUIZ, V. M. Qualidade de vida no trabalho: um desafio no século XXI. In: UNIFAE - Centro Universitário das Faculdades Associadas de Ensino de São João da Boa Vista (vários autores). Pesquisas e temáticas em desenvolvimento sustentável. Itu: Ottoni, 2007.

SANT'ANNA, A. S.; KILIMNIK, Z. M.; MORAES, L. F. R. Antecedentes, origens e evolução do movimento em torno da Qualidade de Vida no Trabalho. In: SANT'ANNA, A. S.; KILIMNIK, Z. M. (orgs.) Qualidade de vida no trabalho: abordagens e fundamentos. Rio de Janeiro: Elsevier; Belo Horizonte, MG: Fundação Dom Cabral, 2011.

SILVA, K. A.; PEDROSO, B.; PILATTI, L.A. Qualidade de vida no trabalho e sociedade pósmoderna: construção de um instrumento de avaliação. Revista Eletrônica FAFIT/FACIC, v. 4, n. 2, p. 11-25, 2010.

VERGARA, S. C. Projetos e relatórios de pesquisa em Administração. São Paulo: Atlas, 2003.

WALTON, R. E. Quality of working life: what is it? Sloan Management Review, v. 15, n. 11, p. 11-21, 1973. 